\title{
An Empirical Study on Inter-Organisational Network Structures for Connected Cars
}

Fumihiko Isada

Kansai University, Japan

\begin{abstract}
This study aimed to empirically examine whether rapid changes in the technological environment affect inter-organisational relationships. In the automotive industry, the advantage of cohesive and vertically integrated inter-organisational relationships has been pointed out conventionally. The development of connected cars, which can communicate bidirectionally with other systems outside the car, is eliciting significant changes in the traditional automotive products and the industry structure - changes generally associated with the automotive product structure moving closer to IT products. The aim was to empirically clarify what kind of network structure of inter-organisational relations firms are growing with changes in industrial structure by creating a database of actual firm behavior. The database was analyzed using the social network analysis method, and the characteristics of growing firms' organisational network structure were extracted. Besides the traditional integrated inter-organisational relationships, the results show that there is a mixture of interorganisational relationships with different characteristics of growth firms.
\end{abstract}

Keywords: inter-organisational network; business ecosystem; platform leadership; social network analysis; connected car

JEL classification: $\mathrm{O} 32$

Paper type: Research article

Received: Mar 08, 2021

Accepted: May 27, 2021

DOI: 10.54820/NVFQ4387 


\section{Introduction}

The network structure of firms' inter-organisational relationships varies, depending on the industry characteristics and product structure to which the firms belong. Technological differences affect industry characteristics and product structures, such as component composition, and the optimal inter-organisational network also is likely to differ in each case. The traditional automobile industry has functioned under a network structure of closed and strong inter-organisational relationships with a specific group of firms. To exchange important technical, development, and design information with partners in a flexible manner and prevent leakage of such information to non-partners, each Automobile manufacturer has strengthened its human and capital relationships with a limited number of specific partners, forming a strong inter-organisational network over the long term.

However, the IT industry has opted for a relatively open, ad hoc, and weak network structure of inter-organisational relationships. Computers and the Internet have standardized interfaces that are available for free or for a fee, and they have become increasingly modular (Ulrich, 1995) in their components. The IT industry has seen the emergence of so-called platformers (or platform leaders) that specialize in a particular function, expand their inter-organisational networks to include a variety of firms, and become dominant in certain areas. And now, the development of CASE (Connected, Autonomous, Shared, and Electric vehicles) in the automotive industry is eliciting significant changes in the traditional automotive products and industry structure - changes generally associated with the automotive product structure moving closer to IT products. A connected car is a car that can communicate bidirectionally with other systems outside of the car (Turner, 2011). This allows the car to share internet access, and hence data, with other devices both inside and outside the vehicle. Connected refers to the car's transformation into an information and communication device. Iansiti et al. (2017) foresee a shift in the industry structure of connected cars, with Google and Apple as platformers and automakers as complements.

This study aimed to empirically clarify what kind of network structure of interorganisational relations firms are growing with changes in industrial structure by creating a database of actual firm behavior. However, changes in each CASE vehicle's industrial structure are expected to be different; therefore, it is necessary to separate the changes in each of the four areas and analyze them separately. For example, Fujimoto (2020), from the perspective of product architecture, categorized ICT-related companies, whose industrial structure is open and modular, as the 'sky' and the existing automobile industry, whose architecture is mainly closed and integral, as the 'ground': 'The Connected is likely to be led by Google, Apple, etc., in the "sky." Electric vehicles, on the other hand, will be led by automotive and power electronics companies on the "ground," because they understand the laws of physics. The Connected Car and car-sharing sector will entail a complex interplay of competition and collaboration between ICT companies with network power and car manufacturers with automotive asset knowledge. In this study's analysis, among the four CASE areas, the focus is on Connected Car, which is pointed out to be the most likely to shift to the 'sky' first. Then, the group of companies involved in connected cars was divided into several types and the relationships between organizations were analyzed.

For the organization of this research, a database was constructed by extracting press releases and English-language newspaper articles to include as much information as possible from all over the world, including the latest information. The database then was analyzed using the social network analysis method. For the inter- 
organisational network structure analysis, each company was viewed as a node in the network. Using social network analysis, various indicators that show the characteristics of the network's structure can be calculated (Borgatti et al., 2002).

\section{Previous Research}

Social network analysis is a method of quantitatively analyzing the impact of the structure of the networks in which people and organizations are embedded on their behavior and performance (Granovetter, 2005). Granovetter (1973) argued that for diffusion across a network, weak ties are the most valuable. Specifically, more novel information flows to individuals through weak rather than strong ties. As close friends tend to move in the same circles, the information they receive overlaps considerably with what is already known. Acquaintances, in contrast, know people in other circles and thus receive more novel information.

The egocentric network is a self-centered network structure. Organizations are connected in a broad network structure, but each organization is connected to the surrounding organizations in different ways. The main interest of egocentric network research is the difference in organizational performance due to the network structure around the organization. The study of ego-centric network structures focuses on the question of triadic closure, i.e. whether the nodes to which the ego node is directly connected are also connected. When there is no direct connection between those who are connected to the ego, a structural hole is said to exist between them (Burt, 1992). On the other hand, if two people who are connected to the ego are themselves connected, the three people (the triad) are described as closed. The high network density of the ego represents the degree to which the ego network triad is closed (Phelps et al., 2012). Bridging Ties (Burt, 2004) are defined as ties that connect separated individuals and groups. Its structural features include many bridge ties and a wide range of connectivity; these can be analyzed by indexes such as the number of intervening ties and structural holes. Bridging Ties' strength lies in the widespread dissemination of new, formal, and heterogeneous knowledge, and it is easily linked to radical innovation.

Among the indicators of network structure, centrality is one of the most commonly used indicators in network analysis. It is an indicator of the degree to which each node in the network is in a central position in the network. There are various ways of thinking about what constitutes a central position, and various indices of centrality have been proposed (e.g. Bonacich, 2007; Freeman et al., 1979). For example, there is degree centrality, a centrality index that defines the degree of a node as its centrality. Here, the degree is the number of ties connected to the node. In other words, the more ties a node has with other nodes, the more central a position it is considered to have. In ego networks, the size of the ego network is of equal value. Betweenness centrality is a centrality index based on the degree to which a node mediates the relationship between other nodes. It is defined as the proportion of the node's presence on a line connecting pairs of other points. The more a node mediates the relationship between other nodes, the more central it is considered to be. A node with high betweenness centrality is considered to be able to control the relationships and information between other nodes.

\section{Research Hypotheses}

One of the most important issues in previous studies was the inter-organisational network's breadth. With the change to Connected Car, companies in the automotive industry need to work on various new technological elements that have not been covered in the past. Furthermore, automotive-related companies are 
required to work together with not only the automobile industry, but also various companies, such as media, distribution, and service companies. It is assumed that the wider the inter-organisational network, the more the companies will grow. Degree centrality is one of the network indicators of network size.

In addition, a company's growth index can be sales, profit margin. However, it is difficult to extract sales and profits related to Connected Car separately from financial statements, etc. Therefore, in this study, it was decided that the ecosystem's growth was viewed as a proxy variable for the degree of growth for firms. As mentioned earlier, in the area of Connected Car, the degree to which the ecosystem surrounding a company is expanded significantly impacts the company's growth potential and is assumed to be an important strategic goal of each company. The growth of the ecosystem of each company was to be evaluated in expansion speed of the size of each node ego network. Therefore, the following hypothesis is proposed:

- Hypothesis 1: In a connected car inter-organizational network, the larger the degree centrality of a node, the faster the network size grows.

Another important indicator in social network analysis, as mentioned in the previous section, is the centrality of each node in the ego network. This is related closely to whether inter-organisational relationships are horizontal divisions of labor or vertically integrated. The question is whether the change to Connected Car will bring automobiles' product structure closer to the modularity of computers. In computers and IT products, modularity promotes innovation in an ecosystem (Iansiti et al., 2004). In such a horizontal division-of-labor industrial structure, the ecosystem's core firms are platformers - i.e., platform leaders (Gawer et al., 2002). Platform leaders can grow by expanding the entire ecosystem through collaborations with companies that supply complementary products and services around their products and technical standards. Among the indicators of centrality in the social network analysis, ego betweenness can be considered to be proxy variables for the egonetwork structure of inter-organizational relations in platform companies. Here, network size affects this index; for example, if the ego network's size increases, the number of intermediaries may increase naturally. So nEgoBetweeness, which is an index showing the normalized ratio, was considered more desirable as a measure for comparison. Also, if the value of the constraint index is small, the network around the firm is sparse, indicating that the firm plays the role of a bridge in the ego network. Therefore, whether a company is a platform company is evaluated using the inverse of the degree of constraint.

In addition, as the network structure among organisations becomes platformbased, companies that complement platform companies also are expected to grow, along with the entire ecosystem's growth. Among the indicators of centrality in the social network analysis, eigenvector centrality can be considered to be proxy variables for the ego-network structure of inter-organizational relations in complementary firms that collaborate with central platform firms. Eigenvector centrality is not a simple centrality, but one that is loaded based on collaborations with highly centric firms. The following hypotheses are proposed:

- Hypothesis 2a: In a connected car inter-organizational network, the larger the $n E g o B e t w e e n e s s$ of a node, the faster the network size grows.

- Hypothesis 2b: In a connected car inter-organizational network, the smaller the constraint of a node, the faster the network size grows.

- Hypothesis 2c: In a connected car inter-organizational network, the larger the Eigenvector centrality of a node, the faster the network size grows. 
However, as an opposing hypothesis to Hypotheses 1 and 2, the network structure of inter-organisational relations may remain unchanged. That is, open interorganisational networks and platforms may not necessarily promote companies' growth. According to previous studies on modularity traps (Chesbrough et al., 2001), if the change to Connected Car is in the early stage of a large technological lifecycle, individual companies will integrate their product structures vertically, and inter-organisational networks' structures will become closed and dense. Wessel et al. (2016) provided a case study of electric vehicles and pointed out the importance of strengthening interdependence in business ecosystems when innovative technological evolution occurs. In the current situation, the industry as a whole remains in a chaotic state, and it is expected to take several decades for the dominant design to be shifted (Fujimoto, 2020). Among the indices of social network analysis, ego network density and closeness centrality are considered as the indices which show the characteristics of the inter-organizational relationship of the growing companies in the conventional automobile industry. The higher the density value, the more closely connected the nodes are in the ego network. It is estimated that the value will increase as the grouping of companies progresses Closeness centrality is a centrality that is loaded based on the relationship between nodes that are close in distance and is factored in to indicate the number of companies with close relationships. The following hypothesis is proposed:

- Hypothesis 3a: In a connected car inter-organizational network, the larger the ego network density of a node, the faster the network size grows.

- Hypothesis 3b: In a connected car inter-organizational network, the larger the closeness centrality of a node, the faster the network size grows.

\section{Research Methodology}

\section{Research Data}

As a data-collection source to verify each of the aforementioned research hypotheses, this study used data from newspaper articles and corporate press releases, through which it is possible to collect comprehensive, integrated, and timely data on the relationships among many firms. For example, in the case of data on mergers and acquisitions, more accurate data can be collected by using investor relations materials, such as companies' annual reports. However, it is not always the case that a clear business relationship exists between the companies that comprise the ecosystem. Jacobides (2019) cites the relationship between Apple and 2 million app developers for Apple's iPhone as an example of the relationships that comprise a business ecosystem. In this study, these loose relationships between firms are also important in analyzing the firms' growth and were included in the analysis. Newspaper articles and press releases are viewed as the rare media through which to collect data on such diverse relationships comprehensively and globally.

Of course, one of the problems with newspaper article data is that they are biased due to story selection at the article stage, depending on individual companies' editorial policies. This research searches various newspaper articles from all over the world, to collect data as comprehensively as possible. Specifically, the Lexis Advance database, created by LexisNexis of the US, was used as the data source. The author's university has a contract with Lexis Advance, which allows for full-text searches and browsing of newspaper articles from over 100 of the world's leading newspapers, including the New York Times, Los Angeles Times, Le Monde, and others.

As for the extraction conditions for the articles, articles containing the search term 'connected car' (not case-sensitive) and containing more than one company were 
extracted. For the target period of extraction, the period for evaluating the growth of companies was set at five years, and articles from 2019 and 2015 were extracted. As extraction conditions, the language was English, and the publication type was limited to newswires and press releases only, excluding other data such as journal articles, theses, and blogs to avoid data duplication.

\section{Analytical Method}

After constructing the database for the analysis as described above, the analysis of the database was conducted through the following steps to verify each hypothesis.

The database for the analysis was based on newspaper articles, and the notation of company names, product names, industry names, locations, and inter-firm relationships is not consistent. For example, some company names are complete, while others include abbreviations, common names, or notation errors. Therefore, all the words and compound words in the entire database were extracted using textmining techniques, and the notations were unified or grouped. Company names were extracted, compared, and transformed to match perfectly, as they are key items in the analysis described below. For regions and inter-firm relationships, categorization was conducted based on the extracted words and phrases' semantic content. KH Coder (Version 3) was used for text mining, and categorization was done using coding rules, a KH Coder function (Higuchi, 2016; 2017).

The extracted relationships between companies were narrowed to the relationships that correspond to the business ecosystem, which is the target of this study's analysis. Considering that the relationships among firms in the extracted articles include competitive and litigation relationships, the relationships were limited to those corresponding to the following. Extracted relationships include, first, strong relationships based on capital relationships, such as acquisition, investment, funding, parent company, and subsidiary. Second, alliances and partnerships, such as partnership, alliance, joint venture, joint development, joint test, cooperative, and support, also were extracted. In addition, mainly transactional relationships in the market, such as client, offer, supply, integration, commitment, and marketing, were extracted.

Next, each company's inter-organisational network structure was analyzed according to each hypothesis based on the database for analysis. In this study, UCINet (Version 6) was used to calculate network indices. In addition, an evaluation index for each firm's degree of growth was calculated. The difference in the size of the ego network from 2015 to 2019 was employed for each firm. To test each hypothesis, a multiple regression analysis was performed with the degree of growth as the dependent variable and the network indicators as the independent variables. SPSS (Version 25) was used for the multiple regression analysis.

As mentioned above, the connected car is composed of components belonging to various industries, such as hardware, software, and services, and the interorganisational relationships among all components may not necessarily be uniform. Therefore, based on the data extracted by the following procedure, the components were exploratively classified into several types, and each type was tested for each of the above hypotheses.

\section{Research Results}

\section{Overview of the Research}

As an overview of the conducted research based on the aforementioned research method, the number of articles extracted was 2,621 in 2015 and 1,861 in 2019, and the number of companies extracted was 1,784, with 18,424 combinations among 
these companies. The firms included non-profit organizations, such as universities and public research institutions. Out of 18,424 combinations of companies, data corresponding to relationships encompassed by business ecosystems was selected as described above, resulting in 4,102 in 2015 and 3,834 in 2019.

Next, an exploratory typology of article data was attempted to analyze the connected car by component. The nouns in the articles were extracted by text mining methods and classified into four categories based on their content. The first category is automotive hardware, such as vehicles and vehicle parts; the second category is connected equipment, such as computer-related equipment, electronic equipment, and communication equipment; the third category is software applications, such as applications, software, and systems; and the fourth category is service, such as services, distribution, and transport. As a result, the number of firms and the number of inter-firm relationships for each category is 756 firms and 6,000 pairs for automotive hardware, 765 firms and 6,067 pairs for connected devices, 416 firms and 3,648 pairs for software applications, and 678 firms and 5,430 pairs for services. The number of firms in each category and the number of inter-firm relationships overlap among the categories. For example, finished automobile product manufacturers appear in articles of all categories. However, they play a relatively central role in articles about the automotive hardware category, while they play a relatively minor role, for example, in articles about the application category.

\section{Results from multiple regression analysis}

In the course of this research, a correlation analysis was first carried out and later a multiple regression analysis, but the results were quite different. From the point of view of robustness of the analysis, the following analysis and discussion are based on multiple regression analysis.

Table 1 shows the standardized regression coefficients from the multiple regression analysis for automotive hardware. Model 1 is the result of forced imputation of all independent variables and Model 2 is the result of stepwise imputation of independent variables. As a result of the analysis, Constraint and Closeness centrality were removed from the analysis due to multicollinearity problems. Of the network indicators, Constraint and Closeness centrality are considered to be similar to nEgoBetweeness and Density, respectively. In the following discussion, these two indices are excluded.

Table 1

Results of multiple regression analysis for Automotive hardware

\begin{tabular}{lcc}
\hline & Model 1 & Model 2 \\
\hline Degree Centrality & $.926^{* *}$ & $.979^{* *}$ \\
nEgoBetweeness & $.519^{* *}$ & $.531^{* *}$ \\
Constraint & -.067 & \\
Eigenvector centrality & $-.298^{* *}$ & $-.320^{* *}$ \\
Density & $.723^{* *}$ & $.721^{* *}$ \\
Closeness centrality & .002 & .330 \\
Adjusted R-square & .330 & $<.001$ \\
\hline Significance probability & $<.001$ & \\
\hline
\end{tabular}

Note: ${ }^{* *} \mathrm{p}<.01,{ }^{*} \mathrm{p}<.05$

Table 2 shows the regression coefficients after standardization from the multiple regression analysis on Connected equipment. Model 1 is the result of forced imputation of all independent variables and Model 2 is the result of the imputation of independent variables stepwise. As a result of the analysis, Constraint and Closeness 
centrality were removed from the analysis because of multicollinearity problems, as in the case of automotive hardware.

Table 2

Results of the multiple regression analysis for Connected equipment

\begin{tabular}{lcc}
\hline & Model 1 & Model 2 \\
\hline Degree Centrality & $.869^{* *}$ & $.923^{* *}$ \\
nEgoBetweeness & $.472^{* *}$ & $.476^{* *}$ \\
Constraint & -.059 & \\
Eigenvector centrality & $-.287^{* *}$ & $-.311^{* *}$ \\
Density & $.688^{* *}$ & $.679^{* *}$ \\
Closeness centrality & -.007 & .280 \\
Adjusted R-square & .280 & $<.001$ \\
\hline Significance probability & $<.001$ & \\
\hline
\end{tabular}

Note: ${ }^{* *} p<.01, * p<.05$

Table 3 shows the standardized regression coefficients from the multiple regression analysis for software applications. Model 1 is the result of forced imputation of all independent variables, while model 2 is the result of stepwise imputation of the independent variables. In the results of the analysis of software applications, as in the previous two analyses, Constraint and Closeness centrality were removed from the analysis due to multicollinearity problems.

Table 3

Results of the multiple regression analysis for software applications

\begin{tabular}{lcc}
\hline & Model 1 & Model 2 \\
\hline Degree Centrality & $.905^{* *}$ & $.951^{* *}$ \\
nEgoBetweeness & $1.099^{* *}$ & $1.145^{* *}$ \\
Constraint & -.054 & $-.280^{* *}$ \\
Eigenvector centrality & $-.267^{* *}$ & $1.441^{* *}$ \\
Density & $1.402^{* *}$ & .264 \\
Closeness centrality & .021 & $<.001$ \\
Adjusted R-square & .261 & $<.001$ \\
Significance probability & & $<$ \\
\hline
\end{tabular}

Note: ${ }^{* *} p<.01,{ }^{*} p<.05$

Table 4 shows the regression coefficients after standardization from the multiple regression analysis for Service. Model 1 is the result of forced imputation of all the independent variables, while model 2 is the result of imputing the independent variables stepwise. In the results of the Service analysis, as in the previous three analyses, Constraint and Closeness centrality were removed from the analysis due to multicollinearity problems.

Table 4

Results of the multiple regression analysis for Service

\begin{tabular}{lcc}
\hline & Model 1 & Model 2 \\
\hline Degree Centrality & $.822^{* *}$ & $.919^{* *}$ \\
nEgoBetweeness & $.645^{* *}$ & $.698^{* *}$ \\
Constraint & -.107 & \\
Eigenvector centrality & $-.259^{* *}$ & $-.299^{* *}$ \\
Density & $.837^{* *}$ & $.867^{* *}$ \\
Closeness centrality & .021 & .291 \\
Adjusted R-square & .293 & $<.001$ \\
\hline Significance probability & $<.001$ & \\
\hline
\end{tabular}

Note: ${ }^{* *} \mathrm{p}<.01, * \mathrm{p}<.05$ 


\section{Discussion}

Based on the aforementioned analysis, each hypothesis was verified. As a result of the analysis, no difference in the relationship between each network index and the degree of growth was observed among components such as hardware and software, so the discussion is based on the entire database.

The first hypothesis concerned network size. Network size is positively related to the degree of growth, indicating that the hypothesis may be supported. A search for companies with a particularly large "degree centrality" in the database for the analysis identified existing car manufacturers such as BMW, Daimler, and Ford, and car parts manufacturers such as Continental, Denso, Harman, and Robert Bosch. These companies are the major players in the traditional automotive business. These existing major companies are trying to continue their traditional business while adapting to new technological changes. They may be growing by retaining their traditional partners, but also by actively adding various partners for new business development, backed by their abundant financial resources. Such a growth pattern can be named as the growth pattern of existing large firms.

The second hypothesis is the mediation hypothesis. The results of the analysis show that the mediation index is positively related to the level of growth and that the hypothesis may be supported. This means that there is a possibility that platform leader firms are growing in the area of connected cars. A search of the database for companies with particularly large nEgobetweeness shows that, in addition to established automakers such as Toyota, there are also large IT platformers such as Amazon, Apple, Google (Alphabet), and Microsoft, telecom equipment companies such as Huawei and Samsung, as well as automotive original equipment manufacturers (OEMs) such as Magna. For example, major car manufacturers such as Toyota are trying to form their platform for connected cars and become the center of collaboration with various services. On the other hand, IT platform providers such as Apple and Microsoft are also expanding their platforms in the automotive space and are competing with automakers for leadership. In the future, IT platforms may become platform leaders in the automotive industry, as they have in many other industries, including computing. It is also possible that OEMs such as Magna will be at the center of inter-organisational relationships in the horizontal division of labor, as Dell once was in IT and Hon Hai Precision Industry is today in smartphones. In the future, the industry structure and inter-organisational relationships in the automotive industry may change significantly, as in the IT industry. Such a growth pattern could be named the platform leader type.

Next, eigenvector centrality indicates the extent to which a firm is connected to a highly centralized firm. The results of the analysis show a negative relationship with the degree of growth, rejecting the hypothesis and suggesting that firms far from the network center may be growing. A search of the database for firms with particularly small Eigenvector centrality reveals connected car service firms such as Gentex and Mojio, as well as telecom equipment and semiconductor manufacturers such as Cisco, Ericsson, and Texas Instruments. These companies are likely to expand their services in the connected car business area while maintaining relative independence from individual existing automakers. Unlike the aforementioned socalled platform leaders, they may be growing in niche business areas. Such a growth pattern can be called a peripheral growth pattern.

The third hypothesis concerns the density and closeness centrality of the network. The results of the analysis show that the index of density may be positively related to growth. On the other hand, in the database for the analysis, not only existing car manufacturers such as Fiat Chrysler and Nissan, but also IT solutions companies such 
as SAP, and telecom operators such as Verizon and Vodafone were searched for as companies with particularly high density. In deriving the hypotheses, existing automobile manufacturers and existing parts manufacturers were assumed, so it cannot be said that the hypotheses were necessarily supported. The nonautomotive firms, such as solution firms and telecommunication firms, have formed their group of firms and are growing. Each is not necessarily a platformer for the entire automotive industry, and their activities may be rather regional, such as the US or Europe, with each regional group of companies providing connected car-related services. Such a growth pattern could be termed a clustered growth pattern.

The discussion so far suggests that the connected car space may be growing with a mix of inter-organisational networks with different characteristics, such as large established firms, platform leaders, periphery, and clusters. It is observed that the traditional inter-organizational relationships in the automotive industry, which have been in place for a long time, are changing due to the radical technological change of the connected car. The changes in industry structure and interorganisational relations due to the connected car are still in progress and may change further. If existing automotive companies are unable to respond to such changes, they may find themselves in an innovation dilemma (Christensen, 1997).

\section{Conclusion}

In the automotive industry, the advantage of cohesive and vertically integrated inter-organisational relationships has been pointed out conventionally. The results of the analysis of a large number of real databases show that the whole of Connected Car does not have the same characteristics of inter-organisational relations, but that growth companies with an inter-organisational network structure of various characteristics coexist. The results also indicate that, possibly, the changes caused by connected cars are driving a change towards a horizontal division of labor in inter-organizational relations, such as the emergence of so-called platform leader firms in the automotive industry.

As the limitations of this research, the industrial structure in this area remains in flux and is expected to change in the future, so the results from this analysis cannot be used as a fixed conclusion. In addition, it cannot be denied that the analysis, based on newspaper article data, may differ from reality. As future research, it is necessary to conduct a continuous analysis while supplementing data with other methods.

\section{References}

1. Bonacich, P. (2007), "Some unique properties of eigenvector centrality", Social networks, Vol. 29 No. 4, pp. 555-564.

2. Borgatti, S. P., Everett, M. G., Freeman, L. C. (2002), Ucinet for windows: Software for social network analysis, Analytic Technologies, Boston.

3. Burt, R. (1992), Structural holes: The social structure of competition, Harvard University Press, Cambridge.

4. Burt, R. S. (2004), "Structural holes and good ideas", American Journal of Sociology, Vol. 110 No. 2, pp. 349-399.

5. Chesbrough, H., Kusunoki, K. (2001), "The modularity trap: Innovation, technology phase shifts, and the resulting limits of virtual organizations", in Nonaka, I., Teece, D. (Eds.), Managing Industrial Knowledge, Sage, London, pp. 202-230.

6. Christensen, C. M. (1997), The Innovator's Dilemma: When New Technologies Cause Great Firms to Fail, Harvard Business School Press, Boston.

7. Freeman, L. C., Roeder, D., Mulholland, R. R. (1979), "Centrality in social networks: II. Experimental results", Social networks, Vol. 2 No. 2, pp. 119-141. 
8. Fujimoto, T. (2020), "Critiquing the recent discussions on manufacturing with questionable evidence", Akamon Management Review, Vol. 19 No. 5, pp. 159-164.

9. Gawer, A., Cusumano, M. A. (2002), Platform leadership: How Intel, Microsoft, and Cisco drive industry innovation, Harvard Business School Press, Boston.

10. Granovetter, M. S. (1973), "The strength of weak ties", American Journal of Sociology, Vol. 78 No. 6, pp. 1360-1380.

11. Granovetter, M. S. (2005), "The impact of social structure on economic outcomes", Journal of Economic Perspectives, Vol. 19 No. 1, pp. 33-50.

12. Higuchi, K. (2016), "A two-step approach to quantitative content analysis: KH Coder Tutorial Using Anne of Green Gables (Part I)", Ritsumeikan Social Science Review, Vol. 52 No. 3, pp. 77-91.

13. Higuchi, K. (2017), "A two-step approach to quantitative content analysis: KH Coder Tutorial Using Anne of Green Gables (Part II)", Ritsumeikan Social Science Review, Vol. 53 No. 1, pp. 137-147.

14. Iansiti, M., Lakhani, K. R. (2017), "Managing our hub economy", Harvard Business Review, Vol. 96 No. 1, pp. 84-92.

15. Iansiti, M., Levien, R. (2004), The keystone advantage: What the new dynamics of business ecosystems mean for strategy, innovation, and sustainability, Harvard Business Press, Boston.

16. Jacobides, M. G. (2019), "In the ecosystem economy, what's your strategy?", Harvard Business Review, Vol. 97 No. 5, pp. 128-137.

17. Phelps, C., Heidl, R., Wadhwa, A. (2012), "Knowledge, networks, and knowledge networks: A review and research agenda", Journal of Management, Vol. 38 No. 4, pp. 1115-1166.

18. Turner, A. (2011), "The Future of the Connected Car", available at: https://mashable.com/2011/02/26/connected-car/ (23 May 2021)

19. Ulrich, K. (1995), "The role of product architecture in the manufacturing firm", Research Policy, Vol. 24 No. 3, pp. 419-440.

20. Wessel, M., Levie, A., Siegel, R. (2016), "The problem with legacy ecosystems", Harvard Business Review, Vol. 94 No. 11, pp. 68-74.

\section{About the author}

Fumihiko Isada works as a professor at the Faculty of Informatics, Kansai University. He graduated from Osaka University, where he got his Ph.D. with the topic "A Study on the business model of Virtual Project Company". His research interests are international corporate strategy and innovation management. The author can be contacted at email isada@kansai-u.ac.jp 